\title{
« Perceptions de justice organisationnelle dans les collectivités locales : quels impacts sur le bien-être psychologique au travail et sur l'intention de quitter des agents »
}

\author{
Amar Fall, Université de Pau et des Pays Adour / E2S, CREG, Chaire Optima \\ Fatéma Safy-Godineau, Université de Pau et des Pays Adour / E2S, CREG, Chaire Optima \\ David Carassus, Université de Pau et des Pays Adour / E2S, CREG, Chaire Optima
}

Résumé : Cette recherche examine l'effet différencié des quatre dimensions de la justice organisationnelle perçue sur le sentiment de bien-être et sur l'intention de quitter des agents des collectivités locales. Les résultats issus de la méthode des équations structurelles sur un échantillon de 924 agents montrent que, lorsque ces derniers ont le sentiment d'être traités de manière juste et équitable, ils sont plus à même à manifester un meilleur état de bien-être au travail. En revanche, lorsqu'ils ont le sentiment d'être traités de manière injuste, leur intention de quitter la collectivité est plus palpable. Les résultats révèlent aussi que les dimensions de la justice ne sont pas toutes, associées au sentiment de bien-être des agents et à leur intention de quitter leurs collectivités. Lorsqu'elles sont simultanément prises en considération, certaines formes de justice ont un plus fort poids explicatif que d'autres.

Mots clé : justice organisationnelle, bien-être psychologique, intention de quitter, collectivités locales.

\begin{abstract}
This research examines differentiated effect of the four dimensions of organizational justice on feelings of well-being and intention to quit local communities agents. The results from structural equations method on 924 agents show that when agents are feeling to be treated in a fair and equitable way, they are more likely to show a better state of well-being at work. On the other hand, the feeling of being treated unfairly, their intention to quit the community is more palpable. The results also indicate that not all dimensions of organizational justice are associated with agents' feeling of well-being and their intention to quit their communities. When all the dimensions are taken into consideration, some forms of justice have a stronger explanatory effect than others.
\end{abstract}

Key words: organizational justice, psychological well-being, intent to quit, local communities 
Fall, Safy-Godineau et Carassus, @GRH, 2018

\section{Introduction}

Les administrations locales sont encore aujourd'hui soumises à des défis managériaux majeurs (Ragaigne, 2016). L’héritage de la bureaucratie wébérienne qui a fortement façonné la culture de l'administration publique française connaît ainsi de nombreuses limites (Crozier et Friedberg, 1977 ; Desmarais et Jameux, 2001), imposant d'importants espaces d'innovation en matière de management. Ces espaces d'innovation ont alors récemment amené les gestionnaires des organisations publiques à introduire de nouveaux outils managériaux jusqu'alors inédits dans les services publics. Cette situation a entrainé de nombreux changements qui touchent différents aspects d'une organisation publique, que cela soit en termes de recrutement, d'évaluation, de formation, de gestion des carrières, ou de mécanismes de contrôle des institutions (Fall et Molines, 2016), et qui requièrent l'obtention de compétences managériales par les gestionnaires locaux (Ragaigne, 2016).

Par ailleurs, ces outils managériaux utilisés, parfois adaptés du secteur privé, se heurtent généralement aux principes égalitaristes et statutaires en vigueur dans les services publics (Monjardet, 1996; Molines et Fall, 2016), faisant ainsi émerger de nouvelles problématiques d'équité et de justice qu'il convient d'explorer (Molines et Fall, 2016). En effet, dans les milieux organisationnels, l'efficacité du management est souvent liée à la manière dont les outils mis en place sont déployés entre les membres de l'organisation (Adams, 1965 ; Greenberg, 1987). Lorsque les salariés perçoivent que ces outils ne sont pas appliqués dans les règles de l'art, l'effet escompté sur les attitudes et les comportements des collaborateurs est alors généralement limité. Ce constat amène Greenberg (1990) à noter que " les chercheurs ont depuis longtemps reconnu l'importance des idéaux de justice comme une exigence fondamentale dans le fonctionnement efficace de l'organisation et la satisfaction personnelle des individus (p. 399)». En ce sens, les perceptions de justice ou d'injustice à l'égard des pratiques managériales constituent d'importants déterminants des comportements au travail qui nous semblent utiles d'explorer dans le contexte public, notamment au sein des collectivités locales. De manière complémentaire, l'étude de l'influence de la justice à l'égard des pratiques managériales dans le secteur public permettra aussi d'examiner, en partie, l'efficacité des apports du New Public Management (Hood, 1991) qui s'est progressivement imposé pour une rationalisation des ressources et une efficacité des pratiques de management. 
Fall, Safy-Godineau et Carassus, @GRH, 2018

Ainsi, la présente recherche porte sur l'influence des perceptions de justice organisationnelle sur le bien-être psychologique des agents et sur leur intention de quitter leurs collectivités. Dans ce cadre, nous répondrons à la question suivante : Quelle influence exerce la justice organisationnelle perçue sur le bien-être psychologique des agents et sur leur intention de quitter leurs collectivités?

Pour répondre à cette question, nous avons mené une recherche quantitative auprès d'agents de collectivités locales. Cette recherche témoigne de l'importance qu'il faut accorder à un management juste et équitable, et son impact à la fois sur un meilleur état de bien-être au travail et sur l'intention de quitter la collectivité.

Par conséquent, la présente recherche présente un double intérêt. Tout d'abord, c'est l'une des premières en France à examiner empiriquement au sein des collectivités locales, la nature des relations entre les perceptions de justice organisationnelle, le sentiment de bien-être au travail et l'intention de quitter des agents. Il n'existe, à notre connaissance, aucune étude sur ces liens, mis à part les résultats descriptifs fournis par différents baromètres professionnels sur le bien-être dans les collectivités. Les rares études qui existent sur ces relations concernent, pour la majorité, les entreprises du secteur privé ou des organismes du secteur public hors de la France. Ensuite, de nombreux travaux sur la justice organisationnelle ont adopté une vision réductrice de ce concept, pouvant conduire à des relations significatives qui n'existeraient pas si l'ensemble des composantes étaient incluses (Cole et al., 2010 ; Ambrose et Schminke, 2003). Notre recherche tente alors de dépasser cette limite. Ainsi, elle étudie l'effet différencié des dimensions de la justice sur le bien-être et sur l'intention de quitter. En proposant une analyse simultanée des dimensions de la justice organisationnelle, nous serons alors en mesure de présenter des résultats plus complets et d'examiner le pouvoir explicatif des perceptions de justice sur le bien-être des agents et sur leur intention de quitter leur collectivité. Nous pourrons ainsi apporter une réponse aux critiques de la littérature sur le flou autour des processus de justice (Ambrose et Schminke, 2003). Par ailleurs, d'un point de vue managérial, notre étude répond à la préoccupation concrète des organisations locales qui sont amenées à repenser leurs pratiques de gestion des ressources humaines (Simon et al., 2015), notamment en termes d'épanouissement des salariés, de réalisation au travail et de fidélisation. 
Fall, Safy-Godineau et Carassus, @GRH, 2018

Dans ce sens, la première partie de cette recherche vise à définir un cadre théorique pouvant supporter des hypothèses sur les relations entre la justice organisationnelle, le bienêtre psychologique et l'intention de quitter. La seconde partie présentera, elle, l'ensemble de la démarche empirique mise en œuvre pour tester les hypothèses de recherche. La dernière partie proposera, enfin, une discussion des contributions théoriques et managériales de cette recherche, ainsi que les voies futures de recherche.

\section{Présentation du cadre théorique et des hypothèses de recherche}

Nous présentons dans un premier temps notre cadre théorique, puis nos hypothèses de recherche.

\subsection{Un cadre théorique mêlant justice organisationnelle, bien-être et intention de quitter}

La justice organisationnelle correspond à la justice perçue par l'individu sur la manière dont il est traité par son organisation (Adams, 1965 ; Greenberg, 1990). Elle fait référence aux normes sociales qui régissent la distribution des ressources de l'organisation, aux processus et procédures conditionnant cette distribution, et aux relations interpersonnelles qui sont associées à ces procédures et distributions (Bies et Moag, 1986 ; Cropanzano et Greenberg, 1997 ; Folger et Cropanzano, 1998). Elle est composée de quatre dimensions considérées comme conditions sine qua none de succès de toute pratique managériale : distributive, procédurale, interpersonnelle et informationnelle (Adams, 1965 ; Colquitt, 2001 ; Cropanzano et Ambrose, 2001). La justice distributive se réfère au sentiment d'équité perçue à l'égard de la distribution des rétributions matérielles ou socio-émotionnelles. Elle se rapporte à l'évaluation des résultats obtenus par le salarié et des conséquences des décisions d'allocation, surtout en termes de proportionnalité entre les rétributions et les contributions (Adams, 1965 ; Cropanzano et Greenberg, 1997). La justice procédurale exprime, quant-à-elle, le sentiment de justice perçue dans les procédures utilisées pour manager et administrer les ressources de l'organisation (Leventhal, 1980 ; Lind et Tyler, 1988 ; Folger et Greenberg, 1985 ; Folger et Konovsky, 1989). Elle se réfère à la justice perçue des procédures utilisées pour déterminer l'allocation des ressources et donc manager l'organisation (Lind et Tyler, 1988). La justice interpersonnelle fait référence à la justice perçue du traitement interpersonnel reçu par le salarié de la part de ses supérieurs hiérarchiques, lors de la mise en place des procédures ou 
Fall, Safy-Godineau et Carassus, @GRH, 2018

du déploiement du management (Bies et Moag, 1986 ; Bies, 2001). Elle est promue par un traitement digne et respectueux. La justice informationnelle fait enfin référence aux perceptions de justice face aux explications et aux informations reçues dans les échanges interpersonnels, notamment entre supérieurs hiérarchiques et subordonnés. Ces deux dernières dimensions forment la justice interactionnelle (Bies, 2001).

Concernant le bien-être au travail, il est défini comme un état psychologique positif en milieu organisationnel (Diener, 1994). Il dispose de soubassements théoriques, hédoniques et eudémoniques qui englobent des concepts très proches tels que la qualité de vie, la satisfaction, les affects, la santé mentale ou la santé psychologique au travail (Diener, 2000 ; Diener, et al., 1999 ; Gillet al., 2015). Le bien-être hédonique fait référence à la recherche de plaisir et de confort. L'individu se trouve dans un état de bien-être quand les émotions positives l'emportent sur les émotions négatives (Diener et al., 1999 ; Kahneman, Diener et Schwarz, 1999 ; Ryan et Deci, 2001 ; Keyes et al, 2002 ; Ryff et Singer, 2008 ; Deci et Ryan, 2008). Le concept central du courant théorique qui porte cette conception hédoniste du bienêtre est le concept de bien-être subjectif. Il est défini comme l'évaluation tant affective que cognitive qu'un individu fait lui-même de sa vie (Diener, 2000 ; Deci et Ryan, 2008). Cette conception du bien-être au travail est souvent assimilée au concept de satisfaction au travail (Diener, 1984 ; Biétry et Creusier, 2013), qui est définie comme l'état émotionnel positif ou plaisant résultant de l'évaluation faite par une personne de son travail ou de ses expériences de travail (Locke, 1976)

En ce qui concerne le bien-être eudémonique, dans lequel s'inscrit la présente recherche, il fait référence à l'accomplissement de soi, plutôt que la maximisation du plaisir. L'individu se trouve dans un état de bien-être quand il vit en concordance avec lui-même et se réalise dans ce qu'il entreprend (Diener et al., 1999; Kahneman, Diener et Schwarz, 1999 ; Ryan et Deci, 2001 ; Keyes et al, 2002 ; Ryff et Singer, 2008 ; Deci et Ryan, 2008). Le concept central du courant théorique qui porte cette conception eudémonique du bien-être est le concept de bien-être psychologique autour duquel s'article notre problématique. Il est défini comme un fonctionnement psychologique positif (Ryff, 1989; Deci et Ryan, 2008) qui renvoie à l'estime de soi, à la réalisation sociale et à l'intégration sociale (Ryff et Keyes 1995 ; Massé et al., 1998 ; Keyes, 2002). Le bien-être psychologique se caractérise ainsi de relations positives avec autrui, d'un sentiment d'autonomie et de maitrise de son environnement, du sens de la vie et de la croissance personnelle (Ryff, 1989). Il se distingue 
Fall, Safy-Godineau et Carassus, @GRH, 2018

en ce sens de la satisfaction qui est appréhendée comme une réponse comportementale du bien-être psychologique (Delobbe, 2013). Le bien-être psychologique relevant de la conception eudémonique, se présente ainsi comme un antécédent potentiel de la satisfaction qui relève quant à elle, plus du bien-être subjectif et donc de la conception hédonique.

Concernant l'intention de quitter, elle correspond à la dernière manifestation attitudinale qui précède l'acte de quitter l'organisation (O'Neill et Mone, 1998). Elle renvoie à l'attitude conduisant à une rupture de l'appartenance à un système social à l'initiative exclusive du salarié (Colle, 2006 ; El-Akremi et al., 2006 ; Price, 1977). L'intention de quitter constitue ainsi un prédicteur majeur du risque de démission ou de départ volontaire des salariés (Griffeth et al., 2000 ; Tett \& Meyer, 1993). Elle apparait en ce sens comme une variable particulièrement pertinente à examiner dans le contexte des organisations qui sont en quête de performance et de maitrise accrue des effectifs, à l'instar des collectivités territoriales. L'intention de quitter est généralement encouragée par des facteurs personnels et des facteurs liés au milieu de travail comme l'insatisfaction au travail, la charge de travail, l'envie de changer d'emploi, le manque de ressources et de reconnaissance, l'état de santé, la conciliation travail-famille, le désir d'anticiper sa retraite... (Kosloski, Ekerdt et De Viney, 2001 ; Hansez et al., 2005 ; Szubert et Sobala, 2005 ; Siegrist et al., 2007). Par conséquent, ce sont les contextes organisationnels, les changements organisationnels et conjoncturels, les risques psychosociaux inhérents au travail et le manque de reconnaissance qui demeurent très souvent à l'origine des choix de retrait prématuré des salariés. La recherche a d'ailleurs montré que les organisations qui développent des stratégies relatives à ces aspects personnels et organisationnels sont plus propices à maitriser l'intention de quitter des salariés (Hansez et al., 2005 ; Hayman, 2005 ; Bertrand et al., 2010).

\subsection{Des variables spécifiques dans le contexte des collectivités territoriales}

Afin de mieux appréhender les variables mobilisées dans le contexte des collectivités territoriales, certains travaux méritent d'être explorés (Catlla et Albanet, 2009; Catlla, Albanet et de Terssac, 2008 ; Guibert, Grolleau et Mergier, 2012 ; Soufflet et Saboune, 2014 ; Ceyzeriat, Caroly et Abord de Chatillon, 2015). Ces travaux permettent en effet, de ressortir davantage les spécificités de la justice, du bien-être et de l'intention de quitter au sein des collectivités. 
Fall, Safy-Godineau et Carassus, @GRH, 2018

Pour la justice organisationnelle par exemple, Catlla et Albanet (2009) soutiennent, dans une étude réalisée sur les sources des malaises au travail déclarés par les agents territoriaux, que les problématiques de justice et d'équité sont au cœur des préoccupations des agents. En effet, l'existence de normes inadaptées et de règles floues au sein des collectivités sont souvent perçues souvent comme contraignantes (Catlla et Albanet, 2009). Certains agents voient leurs activités normées par des procédures strictes auxquelles ils sont obligés de se soumettre ou se retrouvent quelque fois entre des injonctions contradictoires (Catlla et Albanet, 2009 ; Catlla et al., 2008 ; Guibert, Grolleau et Mergier, 2012). Cette situation de travail empêché ou contrarié crée une insatisfaction, un mécontentement et un sentiment d'injustice à l'égard du management. Le sentiment des agents de ne pas comprendre les choix managériaux les plonge ainsi dans le désarroi. Guibert, Grolleau et Mergier, (2012) parlent d'ailleurs de paralysie managériale que subissent les agents de la fonction publique territoriale. Selon ces auteurs, cette paralysie suscite chez les agents une incompréhension et un sentiment d'injustice, notamment à l'égard de l'attribution des ressources. Le management dans les collectivités territoriales souffre d'un déficit de réactivité et de lisibilité dus à des prises de décisions très centralisées, avec un manque d'échanges et de communication (Guibert et al., 2012). Les agents ne comprennent plus les règles qui régissent leur carrière : primes, avancements, affectations, mobilité. En effet, l'incohérence dans les procédures management, notamment entre les notations attribuées par la hiérarchie et les personnes promues, révèle un vrai souci d'équité selon ces auteurs. Par conséquent, l'opacité des règles au sein des collectivités territoriales génère des malaises au travail qui peuvent entraîner de la frustration, de la colère, de la lassitude et un sentiment d'injustice (Guibert et al., 2012 ; Catlla et Albanet, 2009 ; Dubet, 2006). Selon Catlla et Albanet (2009), le point de vue exprimé par les agents ne concerne pas le système promotionnel en général, mais la manière dont les règles sont appliquées : les agents revendiquent que les règles soient appliquées de manière équitable.

Concernant le bien-être au travail des agents, il semble fortement altéré par les nombreuses pressions que subissent les organisations publiques, résultant des restrictions budgétaires et d'une quête de maîtrise accrue des effectifs (Abord de Chatillon et Desmarais, 2012 ; Rossano Abord de Chatillon et Desmarais, 2015). Les collectivités territoriales sont dans une recherche d'optimisation qui entraine des changements permanents et qui affecte l'activité des managers de proximité et celle des agents (Ceyzeriat, Caroly et Abord de Chatillon, 2015 ; Guibert, Grolleau et Mergier, 2012). Le sentiment d'absence de repères et de 
Fall, Safy-Godineau et Carassus, @GRH, 2018

soutien que perçoivent les agents, conjugué au manque de possibilité d'action des managers de proximité provoque une paralysie managériale (Guibert, Grolleau et Mergier, 2012) qui pourrait impacter l'épanouissement professionnel des agents. D'ailleurs, le baromètre $2018 \mathrm{du}$ bien-être dans la fonction publique territoriale ${ }^{1}$ fait état d'une dégradation de la qualité de vie dans le contexte professionnel territorial. Il souligne que le bien-être des agents est surtout compromis par des insuffisances managériales et un manque de prévention. L'insatisfaction à l'égard des pratiques de management peu appréciées, le manque de reconnaissance de la part des supérieurs hiérarchiques et des collègues, le stress accru et la fatigue nerveuse sont autant de facteurs qui empêchent l'épanouissement professionnel des agents au sein des collectivités territoriales.

En ce qui concerne l'intention de quitter, Catlla, Albanet et de Terssac (2008) soulignent que les causes potentielles des comportements de retrait prématuré au sein des collectivités territoriales sont provoquées par les pratiques opaques en terme de gestion des ressources humaines (recrutement, promotion, formation). Fondées pour la plupart sur le relationnel de l'agent, ces pratiques constituent un réel problème dans les collectivités territoriales selon ces auteurs. Elles entrainent souvent des tensions ou des situations pouvant engendrer chez les agents qui subissent le système une incompréhension et de la frustration pouvant amener certains d'entre eux à quitter la fonction publique territoriale. Selon Catlla et al., (2008), parmi même les agents qui restent, bon nombre sombre dans une forme de lassitude, qui à terme pourrait réveiller chez eux une intention de partir. A cela s'ajoutent les attitudes et commentaires déplacés que subissent certains agents, conjuguées à des infrastructures parfois inadaptées à la cohabitation entre hommes et femmes qui renforce la segmentation sexuée des personnels (Catlla et al., 2008). Dans une autre étude réalisée auprès de 44 agents territoriaux (Soufflet et Saboune, 2014) constatent que la violation du contrat psychologique, qui lie l'agent à sa collectivité, encourage fortement les comportements de retrait et l'intention de quitter des agents, sans départ effectif. Lorsque la collectivité ne tient pas une ou plusieurs de ses obligations vis-à-vis des agents, cela entraine une rupture du contrat psychologique (Morrison et Robinson 1997) et provoquerait des dysfonctionnements organisationnels qui entraineraient un attachement négatif, une désaffection des agents envers la collectivité et une intention de quitter.

\footnotetext{
${ }^{1}$ Baromètre bien-être au travail 2018 «La Gazette » et « Mutuelle National Territoire - MTN »
} 
Fall, Safy-Godineau et Carassus, @GRH, 2018

1.3. Des hypothèses de recherche faisant le lien justice organisationnelle, comme variable explicative, et bien-être / intention de quitter comme variables expliquées

La littérature permet, tout d'abord, d'établir l'existence de liens entre la justice organisationnelle et le bien-être au travail. De nombreuses recherches ont ainsi montré que la justice est positivement associée au bien-être psychologique des employés (Heponiemi, Kuusio, Sinervo, et Elovainio, 2011; Kausto, EloLipponen et Elovainio, 2005 ; Gillet et al., 2015). A titre d'exemple, dans leur étude réalisée auprès 2569 médecins finlandais travaillant dans les secteurs privés et publics, Heponiemi et al. (2011) ont montré que la justice organisationnelle perçue était positivement corrélée avec le sentiment de bien-être des médecins. En revanche, les niveaux de corrélation sont plus forts chez les médecins du secteur privé par rapport à ceux du public. Ainsi, selon Heponiemi et al., (2011), les médecins du privé manifestent un meilleur état de bien-être, en raison notamment d'une perception de justice organisationnelle plus élevée dans leur secteur. Selon ces auteurs, les organismes de santé publique devraient alors investir des efforts pour accroître l'équité dans leurs organisations et améliorer le bien-être de leurs employés. Dans le même sens, l'étude de Kausto, EloLipponen et Elovainio, (2005), auprès de 1443 employés finlandais du secteur technique municipal, établit que les agents qui perçoivent leur organisation comme injuste sont plus exposés à l'épuisement émotionnel, aux symptômes de stress et au mal-être au travail. Inversement, ceux qui perçoivent leur organisation comme juste et équitable sont plus épanouis et présentent un meilleur état de bien-être au travail. Plus récemment, Gillet et al., (2015) montrent, eux, à travers une étude réalisée auprès de 839 salariés québécois, inscrits à l'Ordre des conseillers en ressources humaines agréés, que la justice procédurale et la justice distributive sont positivement liées au bien-être. De nombreux autres travaux ont également révélé des résultats similaires (Knight et Haslam, 2010 ; Wood et al., 2012). Ces premières observations nous amènent alors à formuler l'hypothèse suivante :

Hypothèse 1. La justice organisationnelle perçue exerce un effet positif sur le sentiment de bien-être des agents des collectivités locales.

Ensuite, concernant l'intention de quitter, elle est communément considérée comme le résultat d'une évaluation réfléchie de la justice de l'organisation (El-Akremi, Nasr et Camerman, 2006). Elle peut être une réaction «à chaud» motivée par un traitement 
Fall, Safy-Godineau et Carassus, @GRH, 2018

interpersonnel injuste de la part des figures d'autorité ou par une rétribution insatisfaisante (Colquitt et al., 2001). Dans ce sens, de nombreux travaux établissent l'existence d'un lien négatif entre la justice organisationnelle perçue et l'intention de quitter l'organisation (CohenCharash et Spector, 2001; Colquitt et al., 2001 ; Gillet et al., 2015). Par exemple, Gillet et al. (2015) soutiennent que si les employés estiment que le traitement qu'ils reçoivent dans leur organisation n'est pas juste, ils n'auront pas l'intention de rester définitivement au sein de celle-ci, et chercheront une meilleure reconnaissance dans une autre organisation (Adams, 1965). D'autres auteurs, comme Materson et al., (2000) révèlent, à travers une étude sur 651 salariés en formation continue, dans une grande université nord-est américaine, que la justice procédurale et la justice interactionnelle sont d'importants déterminants négatifs de la volonté des employés de quitter l'entreprise. De la même manière, Dailey et Kirk (1992) soulignent au travers d'une étude sur 192 salariés d'un hôpital public nord-américain que l'intention de quitter est plus fortement et négativement affectée par le niveau de justice interactionnelle. Dans une étude sur le changement organisationnel, Kickul et al., (2002) constatent, eux, que la justice procédurale et la justice des figures d'autorité ou interactionnelle influencent similairement et de manière négative l'intention des employés de quitter l'entreprise suite à un changement majeur. D'autres recherches soulignent également que la justice distributive n'est pas le déterminant le moins fort, parmi les dimensions de la justice, de l'intention de quitter (Roberts et al., 1999 ; Brotheridge et al., 2003). Elle a alors des contributions uniques dans l'explication des intentions de retrait et de départ et, ce, au-delà même des effets de la justice procédurale (Brotheridge et al., 2003). De manière complémentaire, l'étude d'Aryee et al. (2002), auprès de 153 employés du secteur public indien, montre que la justice distributive est plus fortement corrélée avec l'intention de quitter que les dimensions procédurale et interactionnelle. Enfin, la méta-analyse de Cohen-Charash et Spector (2001) souligne des corrélations négatives identiques entre la justice distributive et procédurale, et l'intention de quitter. Ces observations nous conduisent à formuler l'hypothèse suivante :

Hypothèse 2. La justice organisationnelle perçue exerce un effet négatif sur l'intention de quitter des agents des collectivités locales.

Pour affiner davantage ces deux hypothèses, un consensus a été établi sur le fait qu'une compréhension complète des dynamiques de la justice nécessite la prise en compte simultanée de ses différentes composantes et de leurs interactions (Molines et Fall, 2016 ; Cole et al., 
Fall, Safy-Godineau et Carassus, @GRH, 2018

2010 ; Ambroseet Shminke, 2003). Cela permet, en même temps, d'évaluer l'effet différencié des différentes formes de justice, d'examiner leur pouvoir explicatif, mais aussi de dépasser les limites des travaux fondés sur l'étude d'un concept multidimensionnel agrégé (Fall, 2014 ; Molines et Fall, 2016). Ces recommandations nous autorisent alors à décliner nos deux principales hypothèses en quatre sous-hypothèses :

- Hypothèse 1a. La justice distributive perçue exerce un effet positif sur le sentiment de bien-être des agents des collectivités locales.

- Hypothèse $1 \mathrm{~b}$. La justice procédurale perçue exerce un effet positif sur le sentiment de bien-être des agents des collectivités locales.

- Hypothèse 1c. La justice interpersonnelle perçue exerce un effet positif sur le sentiment de bien-être des agents des collectivités locales.

- Hypothèse 1d. La justice informationnelle perçue exerce un effet positif sur le sentiment de bien-être des agents des collectivités locales.

- Hypothèse 2a. La justice distributive perçue exerce un effet négatif sur l'intention de quitter des agents des collectivités locales.

- Hypothèse 2b. La justice procédurale perçue exerce un effet négatif sur l'intention de quitter des agents des collectivités locales.

- Hypothèse 2c. La justice interpersonnelle perçue exerce un effet négatif sur l'intention de quitter des agents des collectivités locales.

- Hypothèse 2d. La justice informationnelle perçue exerce un effet négatif sur l'intention de quitter des agents des collectivités locales.

Au regard de l'ensemble de ces hypothèses et de notre cadre théorique sous-jacent, notre modèle de recherche est le suivant :

Figure 1.: Présentation du modèle théorique

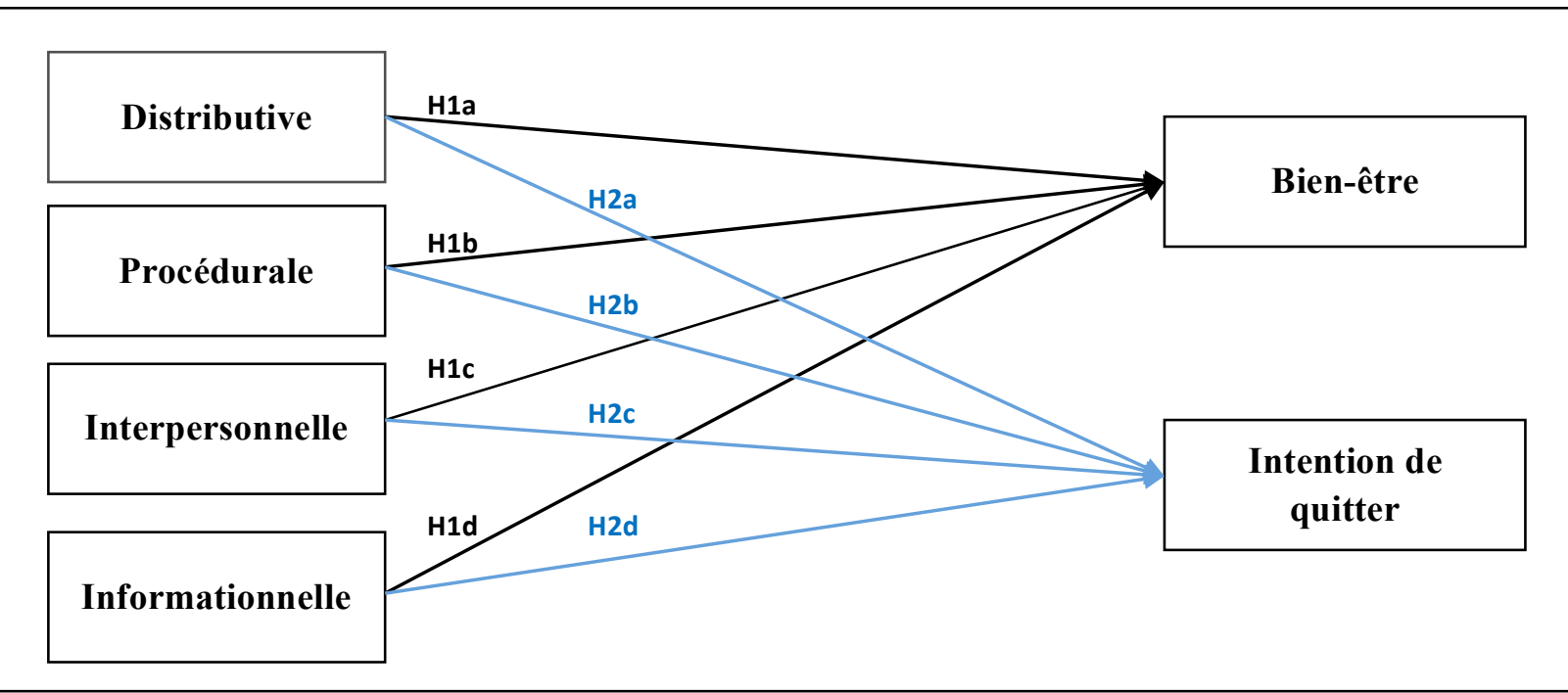


Fall, Safy-Godineau et Carassus, @GRH, 2018

Par la suite, nous testons ce modèle de recherche, sur la base d'une étude quantitative par questionnaire.

\section{Méthodologie et résultats}

Nous présentons dans un premier temps notre méthodologie, sur la base des deux premiers points, concernant notre échantillon et nos instruments de mesure. Dans un second temps, nous présentons nos résultats.

\subsection{Un échantillon de 924 agents}

L'étude empirique a été réalisée en novembre 2017, par la diffusion d'un questionnaire en ligne sur un échantillon de 924 agents de collectivités locales françaises ${ }^{2}$. L'échantillon présenté dans le tableau 1 est composé de 62,2\% de femmes contre 37,8\% d'hommes. Près de $21 \%$ des répondants ont moins de 35 ans, et $36,4 \%$ plus de 50 ans. Environ $89 \%$ des répondants sont des fonctionnaires et $11 \%$ des non titulaires. Ils sont $66,5 \%$ à travailler dans une commune, $17,9 \%$ dans une intercommunalité et $15,7 \%$ dans une région, un département, ou un SDIS. Les répondants sont seulement $0,3 \%$ à travailler dans une collectivité de moins de 499 habitants et environ 62\% à travailler dans une collectivité d'au moins 50000 habitants.

Tableau 1 : caractéristiques de l'échantillon $(\mathrm{N}=924)$

\begin{tabular}{|c|c|r|}
\hline Variables & Modalités de mesure & Pourcentage \\
\hline \multirow{3}{*}{ Genre } & Femme & 62,2 \\
\cline { 2 - 3 } & Homme & 37,8 \\
\hline \multirow{4}{*}{ Age } & Moins de 30 ans & 5,2 \\
\cline { 2 - 3 } & $30-34$ ans & 10,6 \\
\cline { 2 - 3 } & $35-39$ ans & 13,6 \\
\cline { 2 - 3 } & $40-44$ ans & 16,3 \\
\cline { 2 - 3 } & $45-49$ ans & 17,9 \\
\cline { 2 - 3 } & 50-54 ans & 16,8 \\
\cline { 2 - 3 } & 565ans et plus & 19,6 \\
\hline \multirow{4}{*}{ Statut } & Fonctionnaires & 11,1 \\
\cline { 2 - 3 } & Non titulaires & \\
\cline { 2 - 3 } & & \\
\hline
\end{tabular}

\footnotetext{
${ }^{2}$ L'échantillon était composé de 1226 répondants, mais après épuration, nous sommes arrivés à 924 réponses exploitables.
} 
Fall, Safy-Godineau et Carassus, @GRH, 2018

\begin{tabular}{|c|c|c|}
\hline \multirow{4}{*}{ Catégorie } & $\mathrm{C}$ & 21,1 \\
\hline & $\mathrm{B}$ & 15,4 \\
\hline & $\mathrm{A}$ & 28 \\
\hline & Cadre d'emploi & 35,4 \\
\hline \multirow{7}{*}{ Ancienneté } & Moins de 1 an & 6,5 \\
\hline & $1-2$ ans & 8,9 \\
\hline & $3-5$ ans & 15,9 \\
\hline & 6-10 ans & 18,8 \\
\hline & $10-15$ ans & 16,1 \\
\hline & $15-25$ ans & 19,2 \\
\hline & 25 ans et plus & 14,6 \\
\hline \multirow{6}{*}{ Type de collectivité } & Commune & 66,5 \\
\hline & Intercommunalité & 17,9 \\
\hline & Département & 6,7 \\
\hline & Région & 0,9 \\
\hline & SDIS & 3 \\
\hline & Autre & 5,1 \\
\hline \multirow{10}{*}{ Taille de la collectivité } & Moins de 499 habitants & 0,3 \\
\hline & 500-1999 habitants & 1 \\
\hline & 2000-3499 habitants & 1 \\
\hline & 3500-4999 habitants & 1,1 \\
\hline & 5000-9999 habitants & 8,7 \\
\hline & $10000-19999$ habitants & 7,7 \\
\hline & $20000-49999$ habitants & 16,5 \\
\hline & 50 000-99 999 habitants & 37,5 \\
\hline & 100000 habitants et plus & 24,4 \\
\hline & Je ne sais pas & 1,8 \\
\hline
\end{tabular}

2.2. Des instruments de mesures sous forme d'échelles antérieurement validées

Toutes les variables ont été évaluées avec une échelle de Likert à cinq points, allant de 1 «Pas du tout d'accord» à 5 « Tout à fait d'accord». Tous les items en anglais ont été traduits en français par la méthode de back translation (Igalens et Roussel, 1998). Pour la justice organisationnelle perçue, elle est mesurée par 12 items, dont 3 pour chaque dimension, empruntés de l'échelle de Colquitt, (2001) et adaptée au contexte des collectivités locales (ex : ce que j'obtiens de ma collectivité reflète les efforts que j'investis dans mon travail). Cet instrument de mesure de la justice organisationnelle a été choisi surtout pour ses qualités psychométriques avérées. Cette échelle est l'une des plus utilisées depuis le début des années 2000 dans les travaux sur la justice organisationnelle, en raison notamment de ses qualités psychométriques qui ne sont plus à démontrer (Camerman, Cropanzano et Vandenberghe, 2007). Pour le bien-être au travail, il est évalué par 3 items empruntés de l'échelle de Massé et al. (1998), traduite et adaptée en milieu de travail par Gilbert et al., (2011), (ex: je suis bien dans ma peau, en paix avec moi-même). Cette mesure a été choisie pour ses qualités psychométriques avérées, mais également pour sa parcimonie. Bien qu’il existe de nombreuses mesures du bien-être, la conceptualisation synthétique du bien-être validée dans 
Fall, Safy-Godineau et Carassus,@GRH, 2018

la littérature (Massé et al., 1998 ; Gilbert et Dagenais-Desmarais, 2011) est très favorable à son utilisation. Par ailleurs, cette échelle s'inscrit dans une logique eudémonique qui nous permet d'évaluer exactement le bien-être psychologique. De nombreuses autres conceptualisations existant dans la littérature évaluent en effet le bien-être subjectif. Concernant l'intention de quitter, elle est évaluée par 4 items adaptés au contexte des collectivités locales et empruntés de l'échelle de Colle, (2006) (ex : je saisirai la moindre opportunité pour partir de cette collectivité). Cette mesure a été choisie pour sa concordance avec le contexte des collectivités locales. Cette échelle adaptée des travaux de Colle (2006) a été validée par Simon, Carassus et Fall (2014) dans le contexte des collectivités territoriales et répondait donc parfaitement à nos attentes.

Les propriétés des variables, - moyennes, écart types et cohérences internes - exposées dans la matrice des corrélations par la suite, sont toutes satisfaisantes.

\subsection{Des résultats validant partiellement nos hypothèses}

Pour tester notre modèle de recherche, nous avons procédé en plusieurs temps. Tout d'abord, nous avons testé notre modèle de mesure afin de, non seulement, confirmer la bonne validité de construit des questionnaires utilisés, mais aussi de vérifier la qualité de l'ajustement du modèle. Ensuite, nous avons calculé les corrélations entre les différentes variables étudiées pour examiner les risques de multicolinéarité. Enfin, nous avons testé le modèle global en équations structurelles afin de vérifier nos hypothèses de recherche. De nombreux chercheurs (Roussel et al., 2002; Shrout et Bolger, 2002) estiment que la modélisation par équations structurelles offre, en effet, une meilleure analyse simultanée des variables et permet d'éviter les problèmes liés aux erreurs de mesure, à la multicolinéarité et aux relations non linéaires. En intégrant les erreurs de mesure dans l'estimation du modèle étudié, la méthode d'équations structurelles permet alors d'obtenir des coefficients moins biaisés (Roussel et al., 2002).

L'analyse factorielle confirmatoire a été réalisée sur les six variables latentes de premier ordre qui représentent notre modèle théorique (justice distributive, justice procédurale, justice interpersonnelle, justice informationnelle, bien-être et intention de quitter), elles-mêmes définies par les dix-neuf items correspondants. Le tableau 1 présente les résultats de l'analyse factorielle confirmatoire : 
Fall, Safy-Godineau et Carassus, @GRH, 2018

Tableau 1 : Qualité d'ajustement du modèle théorique

\begin{tabular}{|c|c|c|c|c|c|c|c|c|c||}
\hline \hline Indices d'aj. & Chi2 & $\chi$ 2/ddl & GFI & AGFI & SRMR & RMSEA & NFI & NNFI & CFI \\
\hline Modèle théorique & 297.1 & 2.16 & .954 & .936 & .031 & .042 & .968 & .978 & .983 \\
\hline \hline
\end{tabular}

Les résultats mettent en évidence un excellent ajustement des données au modèle théorique. Comme nous pouvons le constater, le Chi2 rapporté à son degré de liberté $\chi^{2 / d d l}$ est égal à 2,17. Ce ratio est satisfaisant car bien inférieur à 5, c'est-à-dire la norme empiriquement fixée à ne pas dépasser. L'indice de résidus SRMR est égal à 0.03 . En règle générale, il est couramment admis que plus l'indice SRMR est proche de zéro, meilleur est l'ajustement. Par ailleurs, l'indice RMSEA est égal à 0.04. Cet indice doit être inférieur à 0.08 pour informer sur un meilleur ajustement. Les autres indices de mesure absolus GFI $=0.954$ et $\mathrm{AGFI}=0.936$ sont également très satisfaisants car supérieurs à la norme communément admise (.90). Les indices de mesures incrémentaux NFI $=0.968, \mathrm{NNFI}=0.978$ et $\mathrm{CFI}=$ 0.983 atteignent aussi les valeurs admises pour offrir un excellent ajustement (Bentler et Benett, 1980; Pedhazur et Pedhazur Schmelkin, 1991; Roussel et al., 2002). Ces résultats apportent du soutien à la validité de construit des questionnaires utilisés dans cette étude.

Au-delà de cette analyse confirmatoire, nous avons ensuite réalisé des statistiques descriptives et étudié les corrélations entre les variables du modèle. Le tableau 2 suivant présente ces résultats.

Tableau 2 : Statistiques descriptives et corrélations entre les variables

\begin{tabular}{|c|c|c|c|c|c|c|c|c|}
\hline Variables du modèle & Moyenne & Ecart-type & 1 & 2 & 3 & 4 & 5 & 6 \\
\hline 1. Justice distributive & 2.83 & 1.06 & $(0,94)$ & & & & & \\
\hline 2. Justice procédurale & 2.84 & 0.92 &, $648^{* *}$ & $(\mathbf{0 , 8 5})$ & & & & \\
\hline 3. Justice interpersonnelle & 3.93 & 0.92 &, $483^{* *}$ &, $594^{* *}$ & $(0,93)$ & & & \\
\hline 4. Justice informationnelle & 3.27 & 1.03 &, $508^{* *}$ &, $652^{* *}$ &, $753^{* *}$ & $(\mathbf{0 , 8 8})$ & & \\
\hline 5. Bien-être au travail & 3.70 & 0.71 &, $208^{* *}$ &, $245^{* *}$ &, $225^{* *}$ &, $244^{* *}$ & $(0,74)$ & \\
\hline 6. Intention de quitter & 2.66 & 1.01 &,$- 332^{* *}$ &,$- 370^{* *}$ &,$- 350^{* *}$ &,$- 381^{* *}$ &,$- 157^{* *}$ & $(0,89)$ \\
\hline
\end{tabular}

$* p<.05 ; * * p<.01$ 
L'analyse descriptive de ces données mérite un premier commentaire. Elle fait ressortir des moyennes supérieures à la moyenne théorique pour certaines de nos variables. Cela indique que les répondants ont été plutôt d'accord avec les énoncés relatifs aux variables proposées. Pour nos variables explicatives, c'est la justice interpersonnelle et la justice informationnelle qui ont les moyennes de réponse les plus fortes. Pour nos variables expliquées, l'intention de quitter connaît des réponses justes inférieures à la moyenne théorique. Le bien-être au travail dispose par contre d'une moyenne bien plus élevée.

Par ailleurs, toutes les dimensions de la justice sont positivement corrélées avec le bien-être : $\mathrm{r}=, 208 ; p=0,000$ pour la justice distributive, $\mathrm{r}=, 245 ; p=0,000$ pour la justice procédurale, $\mathrm{r}=, 225 ; p=0,000$ pour la justice interpersonnelle et enfin $\mathrm{r}=, 244 ; p=0,000$ pour la justice informationnelle. Toutes les dimensions de la justice sont aussi négativement corrélées avec l'intention de quitter : $\mathrm{r}=-, 332 ; p=0,000$ pour la justice distributive, $\mathrm{r}=$ ,370; $p=0,000$ pour la justice procédurale, $\mathrm{r}=-, 350 ; p=0,000$ pour la justice interpersonnelle et $\mathrm{r}=, 381 ; p=0,000$ pour la justice informationnelle. Ces premiers résultats vont dans le sens de nos hypothèses de recherche. La fiabilité des échelles de mesure, calculée avec l'alpha de Cronbach, est, elle, donnée par les valeurs entre parenthèses. Celles-ci sont satisfaisantes, allant de 0.74 à 0.94 .

Afin de statuer définitivement sur le sens des relations observées, nous recourons par la suite à la méthode des équations structurelles. Les analyses sont réalisées à l'aide du logiciel LISREL 9.30. Le tableau 3 présente les résultats des analyses en pistes causales entre la justice organisationnelle, le bien-être et l'intention de quitter. La figure 2 présente, elle, le modèle structurel avec les résultats.

Tableau 3. Résultats des analyses en pistes causales entre la justice, le bien-être et l'intention de quitter

\begin{tabular}{|c|c|c|c|c|c|c|}
\hline \multicolumn{3}{|c|}{ Relations entre les variables } & $\begin{array}{l}\text { Estimations } \\
\text { standardisées }\end{array}$ & $\begin{array}{c}\text { Erreur } \\
\text { standardisée }\end{array}$ & $\begin{array}{c}\text { T de } \\
\text { student }\end{array}$ & $\mathbf{R}^{2}$ \\
\hline Justice distributive & $\longrightarrow$ & Bien-être & .086 & .060 & 1.43 & \multirow{4}{*}{$30 \%$} \\
\hline Justice procédurale & $\longrightarrow$ & Bien-être & .077 & .085 & 0.90 & \\
\hline Justice interpersonnelle & $\longrightarrow$ & Bien-être & $.227 * *$ & .071 & 3.16 & \\
\hline Justice informationnelle & $\longrightarrow$ & Bien-être & $.226 * *$ & .086 & 2.60 & \\
\hline Justice distributive & $\longrightarrow$ & Intention de quitter & -.067 & .061 & -1.09 & $21,9 \%$ \\
\hline
\end{tabular}


Fall, Safy-Godineau et Carassus, @GRH, 2018

\begin{tabular}{|ll|l|l|l|}
\hline Justice procédurale & Intention de quitter & $-.207 * *$ & .088 & $\mathbf{- 2 . 3 4}$ \\
\hline Justice interpersonnelle & Intention de quitter & -.087 & .072 & -1.20 \\
\hline Justice informationnelle & Intention de quitter & $-.166 *$ & .088 & $-\mathbf{I . 8 6}$ \\
\hline${ }^{* *} p<.01 ;{ }^{*} p<.05$
\end{tabular}

Figure 2. Modèle structurel avec les résultats

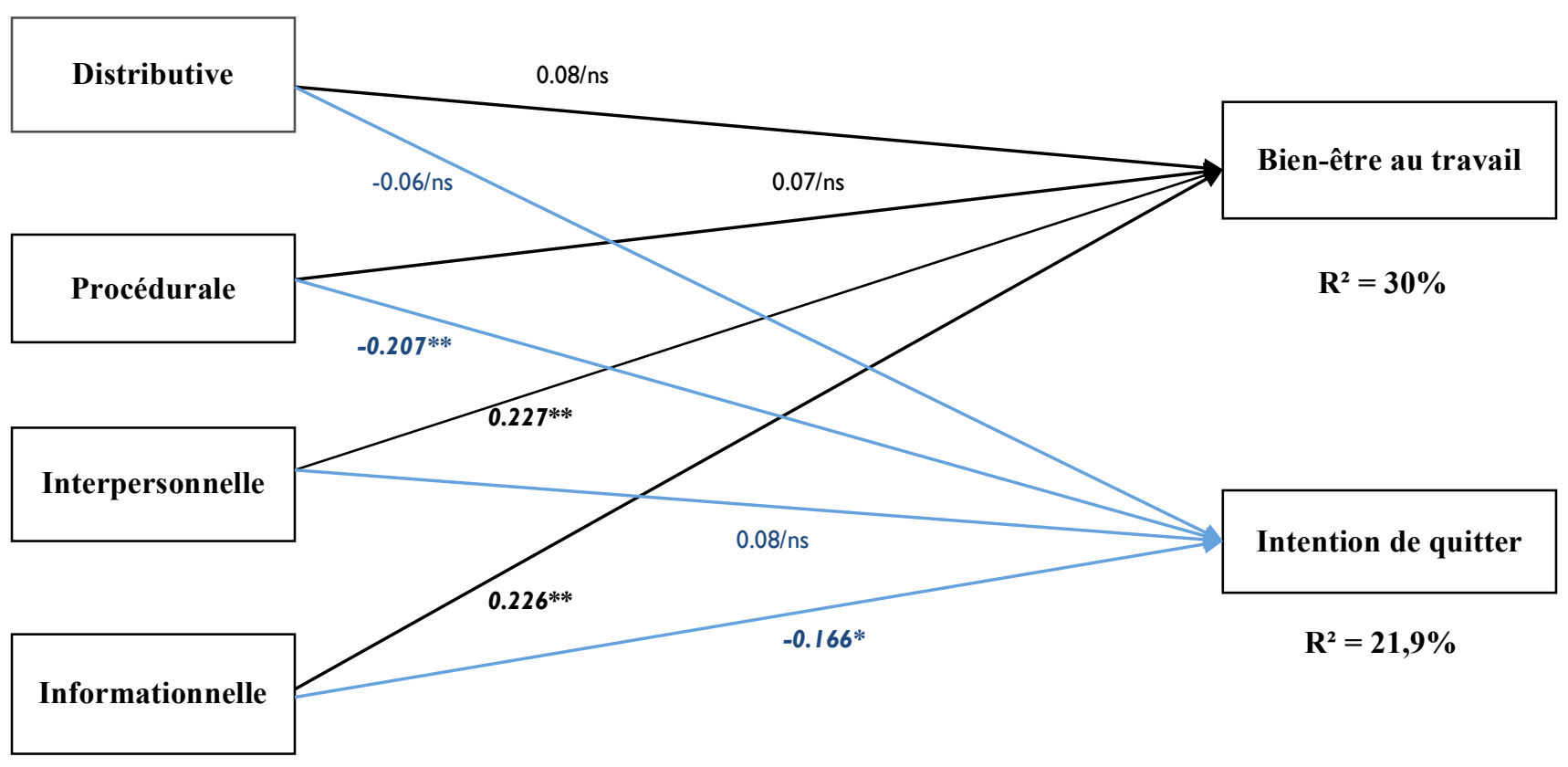

Avant de s'attacher à la validation des hypothèses, nous pouvons remarquer que dans l'ensemble, les données collectées sur notre échantillon s'ajustent parfaitement au modèle structurel $[\chi 2(138)=393,24, \mathrm{p}<.000 ; \mathrm{NNF}=.961 ; \mathrm{NNFI}=.968 ; \mathrm{CFI}=.974 ; \mathrm{RMSEA}=$ $.05 ; \mathrm{GFI}=.941 ; \mathrm{AGFI}=.919]$. En effet, toutes les valeurs sont conformes aux seuils exigés (Pedhazur et Pedhazur Schmelkin, 1991; Roussel et al., 2002).

Les résultats du modèle structurel montrent ensuite que la justice organisationnelle perçue explique au moins $30 \%$ de la variation du bien-être des agents. En revanche, lorsque les quatre formes de justice sont testées simultanément, seules la justice interpersonnelle $\beta=$ $0.227 * *(p=0.002)$ et la justice informationnelle $\beta=0.226^{* *}(p=0.009)$ sont associées au bien-être, validant définitivement les sous-hypothèses H1c et H1d. La justice interpersonnelle perçue, ainsi que la justice informationnelle perçue exercent donc un effet positif sur le sentiment de bien-être des agents des collectivités locales. Les sous-hypothèses H1a et H1b sont par contre, elles, rejetées. 
Fall, Safy-Godineau et Carassus, @GRH, 2018

De la même manière, la justice organisationnelle perçue explique au moins $21,9 \%$ de la variation de l'intention de quitter des agents. Là aussi, seules la justice procédurale $\beta=$ $0.207^{* *}(p=0.001)$ et la justice informationnelle $\beta=-0.166^{*}(p=0.05)$ sont associées à l'intention de quitter, validant les sous-hypothèses $\mathbf{H 2 b}$ et $\mathbf{H 2 d}$. La justice procédurale perçue, ainsi que la justice informationnelle perçue exercent un effet négatif sur l'intention de quitter des agents des collectivités locales. Les sous-hypothèses $\mathrm{H} 2 \mathrm{a}$ et $\mathrm{H} 2 \mathrm{c}$ sont par contre, elles, rejetées.

\section{Discussion des résultats}

Même si de nombreuses recherches ont été consacrées aux facteurs déterminants le bien-être psychologique et l'intention de quitter une organisation, à ce jour très peu d'études empiriques ont porté sur l'influence des perceptions justice organisationnelle dans le contexte des collectivités locales. C'est l'une des raisons pour lesquelles notre recherche se proposait d'apporter une contribution afin combler ce manquement de la littérature. L'impact simultané des quatre formes de justice organisationnelle sur le bien-être et sur l'intention de quitter l'organisation a, ainsi, été testé. Plus précisément, l'analyse de la littérature nous a amené à formuler deux principales hypothèses selon lesquelles les perceptions de justice organisationnelle avaient une influence positive sur le bien-être des agents et négative sur leur intention de quitter leur collectivité. Les données empiriques collectées pour les besoins de cette recherche ont permis de valider toutes les hypothèses, si l'on s'en tient à la matrice des corrélations. Toutefois, l'analyse corrélationnelle n'étant pas toujours suffisante, nous l'avons complétée par la méthode d'équations structurelles afin d'affiner davantage les résultats. Nos analyses complémentaires montrent que toutes les dimensions de la justice organisationnelle n'agissent pas forcément sur le bien-être des agents et sur leur intention de quitter. Lorsque les quatre formes de justice sont mises en évidence simultanément, la justice distributive et la justice procédurale n'ont ainsi aucun impact sur le bien-être des agents. Seules la justice interpersonnelle et la justice informationnelle agissent positivement sur leur bien-être au travail. De même, la justice distributive et la justice interpersonnelle n'ont aucun impact sur l'intention de quitter des agents lorsque les quatre formes de la justice sont simultanément prises en considération. Seules la justice procédurale et la justice informationnelle agissent négativement sur l'intention de quitter. 
Fall, Safy-Godineau et Carassus, @GRH, 2018

D'un point de vue théorique, cette recherche apporte une importante contribution à la littérature. Non seulement elle va dans le sens des travaux antérieurs (Heponiemi, et al., 2011; Kausto, et al., 2005 ; Cohen-Charash et Spector, 2001; Colquitt et al., 2001 ; Gillet et al., 2015), mais elle fournit aussi un éclairage permettant de prendre du recul au regard de certaines digressions présentes dans la littérature. Portant exclusivement sur des salariés du secteur public, en l'occurrence ceux des collectivités locales françaises, cette recherche offre des résultats plus précis quant à la contribution de chacune des dimensions relatives à la justice organisationnelle. A titre d'exemple, si Kausto et al. (2005) estiment que les agents municipaux finlandais qui perçoivent leur organisation comme juste sont plus épanouis au travail et présentent un meilleur état de bien-être, notre recherche permet de préciser que ce sont surtout les perceptions de justice interpersonnelle et informationnelle qui prédisent le sentiment de bien-être des agents. Ces deux dimensions ont le plus fort pouvoir explicatif de leur sentiment de bien-être au travail. Nos résultats remettent aussi en cause ceux de Gillet et al. (2015), en montrant la prééminence des perceptions de justice interpersonnelle et informationnelle dans la prédiction du bien-être en contexte public.

Concernant l'intention de quitter, nos résultats corroborent la plupart des travaux antérieurs (Aquino et al., 1997 ; Materson et al., 2000 ; Kickul et al., 2002). Ils montrent, en effet, que ce sont surtout les perceptions de justice procédurale et interactionnelle qui ont le plus fort pouvoir prédictif négatif de la volonté des employés de quitter l'organisation. En ce sens, nos résultats remettent en question les travaux de Roberts et al., (1999) et ceux de Brotheridge et al., (2003), qui soutiennent que la justice distributive a des contributions uniques dans l'explication des intentions de retrait et de départ et ce, au-delà même des effets de la justice procédurale. Dans le secteur public, notamment au sein des collectivités locales, l'intention de quitter des agents est alors plus fortement liée à l'injustice perçue au regard des procédures ou au regard des informations communiquées.

Le fait que la justice distributive n'a pas d'influence sur le bien-être et sur l'intention de quitter peut-être tout d'abord lié au statut des fonctionnaires territoriaux et aux pratiques RH locales. En effet, le statut dernier protège les agents des problèmes d'équité Ainsi, la distribution des rétributions matérielles ou socio-émotionnelles est souvent identique entre tous les agents, quel que soit les contributions. Elle est relativement homogène au sein d'une même catégorie d'agents avec les mêmes responsabilités et la même ancienneté. De plus, les dispositifs de rémunération à la performance sont peu acceptés et déployés dans les 
Fall, Safy-Godineau et Carassus, @GRH, 2018

collectivités locales alors que cette forme de justice distributive même, questionne le rapport à la rémunération et plus particulièrement une rémunération variable. Ensuite, cette absence d'influence de la justice distributive peut aussi être expliquée par le fonctionnement bureaucratique dans le secteur public (Crozier et Friedberg, 1977). En effet, si la centralisation des décisions, et le fonctionnement cloisonné souvent vertical génèrent des inconvénients importants, notamment en terme de transversalité ou de dynamique collective, ces modes de fonctionnement permettent pourtant de protéger les agents des déséquilibres distributifs. La rémunération des fonctionnaires territoriaux et agents contractuels est déterminée sur des critères relativement fixes (ancienneté, grades, échelons). Ainsi, la justice distributive, même si, prise individuellement, elle est corrélée avec nos variables dépendantes, elle apparaît moins peser que les autres types de justice organisationnelle lorsque toutes les dimensions sont prises en compte en même temps.

Pour mieux comprendre nos résultats, les contributions de la psychodynamique du travail (Dejours, 1992) et de la théorie de l'échange social (Blau, 1964) peuvent aussi être mobilisées. Elles permettent en effet de comprendre davantage la prééminence de certaines dimensions de la justice sur le bien-être et l'intention de quitter des agents.

La psychodynamique du travail (Dejours, 1992) s'attache à analyser l'influence des conduites, des comportements et de la communication des acteurs sur la souffrance et le bienêtre dans le travail. Elle appréhende ainsi le travail comme source de plaisir et de souffrance, mais aussi comme un lieu de production de rapports sociaux, un lieu où l'identité et le positionnement social se construisent par l'entremise, essentiellement, de la reconnaissance (Dejours, 1993). De ce fait, si la hiérarchie n'accepte pas les espaces de discussion, cela conduit à un isolement des individus, source de souffrance au travail (Dejours, 1998). Par conséquent, les interactions, la communication, les espaces de discussion et la négociation de l'organisation du travail, inscrits au cœur des expériences de travail, contribuent directement au plaisir et à la souffrance au travail. Il faut organiser la communication entre les concepteurs du travail et les opérateurs qui l'accomplissent afin d'enrichir l'organisation du travail des opérateurs et éviter toute forme de souffrance (Dejours, 1992 ; Davezies, 1993). Si l'on s'en tient à ces développements de la psychodynamique du travail, on comprend davantage pourquoi les perceptions de justice interpersonnelle et informationnelle agissent mieux sur le bien-être et l'intention de quitter. Un management focalisé sur des relations interpersonnelles justes et sur une qualité optimale des informations communiquées est plus à même à favoriser 
le plaisir ou le bien-être au travail. Un management focalisé sur des relations interpersonnelles injustes et sur un manque de communication favorise davantage de la souffrance au travail, voir une intention de quitter.

Par ailleurs, la théorie de l'échange social (Blau, 1964) offre un cadre d'analyse suffisamment fécond pour comprendre les mécanismes psychologiques organisationnels susceptibles d'expliquer la prééminence des dimensions interactionnelles de la justice sur le bien-être et sur l'intention de quitter. Elle met en évidence l'importance qu'il faut accorder à la qualité des relations interpersonnelles pour favoriser des attitudes positives (comme bienêtre) et éviter des attitudes négatives (comme intention de quitter). Elle considère les organisations, incluant les collectivités territoriales, comme des lieux de transactions mutuelles dans lesquels, la relation managériale demeure la principale ressource échangée (Blau, 1964). Dans cette perspective, les perceptions de justice organisationnelle peuvent engendrer des relations d'échange social (Matterson et al., 2000; Cropanzano et al., 2001), qui constitue la cause proximale pour expliquer les bases motivationnelles des attitudes et comportements des subordonnés (Moorman et Byrnes, 2005; Nasr et al., 2009), y compris leur état de bien-être ou intention de quitter. Selon cette théorie, les effets de la justice sur les attitudes passent avant tout par la qualité de la relation d'échange social. C'est la qualité de la relation d'échange entre le manager et son subordonné qui explique le mieux le lien entre la justice et les attitudes (Konovsky et Pugh, 1994; Rupp et Cropanzano, 2002). Les perceptions de justice interpersonnelle et informationnelle étant étroitement liées à la qualité des informations communiquées et des relations interpersonnelles, seraient plus prégnantes pour la qualité de la relation d'échange et auraient donc plus d'influence sur le bien-être et l'intention de quitter des agents. En effet, lorsque la relation d'échange est fondée sur des règles transparentes, équitables et respectueuses, les subordonnés se sentent plus satisfaits au travail (Ohana, 2012) et sont donc plus épanouis. Ainsi, un traitement équitable est supposé engendrer des relations d'échange de haute qualité qui, par les vertus de la norme de réciprocité, devraient motiver des attitudes positives fonctionnels de la part du salarié (Nasr et al., 2009), tel que le bien-être au travail. Par conséquent, la qualité de l'échange entre le manager semble fondamentale pour comprendre le lien entre la justice organisationnelle et le bien-être. Par ailleurs, selon cette théorie, les individus qui perçoivent un traitement juste de la part de leur organisation se sentent redevables à l'égard de celle-ci, et sont contraints de continuer à y travailler (Blau, 1964). En revanche, lorsque le traitement est perçu comme 
injuste, ces derniers estiment que le contrat psychologique ${ }^{3}$ est rompu, ce qui conduit à une détérioration de la relation de confiance entre le salarié et l'organisation' poussant l'individu à quitter celle-ci (Robinson \& Rousseau, 1994).

D’un point de vue managérial, cette recherche apporte un éclairage non négligeable quant au pouvoir explicatif de la justice organisationnelle perçue. Au regard de nos résultats, cette variable mérite ainsi d'être traitée comme levier potentiel de succès du management. En effet, elle explique au moins $30 \%$ de la variation du bien-être des agents et $21,9 \%$ de la variation de leur intention de quitter. Ces résultats montrent à quel point les managers des collectivités locales devraient accorder plus d'importance à des pratiques managériales justes et équitables. De façon plus concrète et à la lumière de nos résultats, les managers devraient ainsi veiller à ce que les principes de justice interactionnelle soient bien respectés au travers de leurs pratiques de management quotidien afin de favoriser le sentiment de bien-être des agents. Si ce n'est pas déjà le cas, ils devraient alors fonder leurs pratiques sur des relations interpersonnelles et informationnelles justes et transparentes. Pour ce faire, une quête d'impartialité dans les informations communiquées et dans les relations entre supérieurs hiérarchiques et subordonnés, devrait être mise en place. Dans ce sens, les agents doivent être traités avec respect et dignité. Les informations communiquées doivent être claires, sincères et données au bon moment. Les managers doivent aussi adapter leur communication aux besoins spécifiques de chaque agent si possible, et éviter tout type de commentaires déplacés à leur égard. En résumé, d'après les résultats de notre étude, la courtoisie dans les relations interpersonnelles et l'honnêteté dans les informations communiquées devraient être les principes clés qui doivent caractériser un management qui veut encourager le bien-être agents. Les dirigeants et managers des collectivités locales devraient aussi mettre l'accent sur des processus et procédures de management justes pour prévenir ou réduire l'intention de quitter des agents. En plus de l'injustice informationnelle perçue, l'injustice procédurale se présente aussi comme un déterminant potentiel de l'intention de quitter. Il apparait donc tout aussi important d'engager des réflexions au sein des collectivités locales visant à promouvoir davantage un management fondé sur des procédures justes et acceptables. Par exemple, les processus d'évaluation des compétences, les entretiens ante promotion, les grilles

\footnotetext{
${ }^{3}$ Le contrat psychologique correspond à l'entente tacite entre un groupe d'employés et leur contremaitre (Argyris, 1960). Il renvoie à l'ensemble aux promesses et obligations réciproques reliant les employeurs et les salariés (Robinson, Kraatz et Rousseau, 1994),
} 
Fall, Safy-Godineau et Carassus, @GRH, 2018

d'évaluation des performances, les décisions collégiales ou les plans de formation devraient tous respecter les règles de l'art et être utilisés de manière identique pour tous les agents.

Il faut toutefois noter que le manager n'a pas toutes les clés en main pour améliorer la perception de justice ou réduire le sentiment d'injustice que ressentent les agents à l'égard du management. En particulier, il peut être confronté à l'obligation de déployer des pratiques imposées par la hiérarchie (administrative et/ou politique), sur lesquels il n'a que peu de marges de manœuvre. La soumission à l'autorité publique, l'obligation d'exécuter les directives, ainsi que le respect des règles légales et conventionnelles peuvent alors constituer autant d'éléments dont l'incompréhension peut compromettre la perception de justice à l'égard du management.

Ainsi, si cette recherche apporte une contribution significative à la compréhension, théorique et managériale, du bien-être et de l'intention de quitter des agents, elle n'est pas toutefois exempte de limites. Nous en avons déjà identifié certaines, habituelles à un travail quantitatif de nature hypothético-déductive, qui appellent à des prolongements. D'une part, il serait pertinent de réaliser une étude qualitative pour confronter les relations observées au discours des agents eux-mêmes. Cela permettrait d'affiner davantage les résultats. Par ailleurs, étant donné que notre étude est transversale, nos résultats doivent être interprétés avec précaution. En effet, même si l'hétérogénéité de l'échantillon ${ }^{4}$ pourrait être un avantage et permettre une généralisation des résultats, l'aspect temporel du sens des relations n'est pas encore vérifié. Une étude longitudinale permettrait alors, à l'avenir, de pallier à cette limite. Il serait aussi important de croiser nos résultats avec des données relevant de problématiques sous-jacentes, plus que d'actualités au sein des collectivités territoriales, tels que l'absentéisme, les départs effectifs, le turnover, la santé au travail, les risques psychosociaux ou l'implication organisationnelle. Par conséquent, des recherches futures intégrant ces principales limites, permettraient de confirmer nos résultats empiriques.

\footnotetext{
${ }^{4} \mathrm{~L}$ 'hétérogénéité de notre échantillon vient du fait qu'il est composé d'agents appartenant à différentes collectivités locales françaises de taille différente, composées à la fois d'hommes et de femmes, de fonctionnaires et de non titulaires.
} 
Fall, Safy-Godineau et Carassus, @GRH, 2018

\section{Bibliographie}

Adams J.S., 1965, «Inequity in social exchange », in Berkowitz L., Advances in Experimental Social Psychology, volume 2, AcademicPress, New-York, pp. 267-299.

Ambrose, M.L. et Marshall S. (2003) "Organization Structure As a Moderator of the Relationship BetweenProcedural Justice, Interactional Justice, PerceivedOrganizational Support and Supervisory Trust ", Journal of Applied Psychology, 88, p. 295-305.

Aquino, K., Griffeth, R.W., Allen, D.G., \&Hom, P.W. (1997). Integrating justice constructsintothe turnoverprocess: A test of a referent cognitions model. Academy of Management Journal, 40, 1208 -1227.

Aryee, S, Budhwar, P. et Chen, Z.X (2002), Trust as a mediator of the relationship between organizational justice and work outcomes: test of a social exchange model, Journal of Organizational Behavior, 23 (2002), pp. 267-285

Bentler, P. M., \& Benett, D. G. (1980). Significance tests and goodness-of-fit in the analysis of covariance structures. Psychological Bulletin, 88(3), 588-600.

Bies, Robert J. et Joseph S. Moag. (1986). Interactional Justice : Communication Criteria of

Fairness. Dans R.J. Lewicki, Sheppard et M.H. Bazerman, Research on Negociation in Organizations, Greenwich Press.

Bies, R.J. (2001). Interactional (in)justice: The sacred and the profane. In J. Greenberg et R. Blau, P. (1964). Exchange and power in social life. New York: Wiley.

Brislin, W.R. (1970). " Back-Translation for Cross-Cultural Research», Journal of CrossCultural Psychology, 1970, 1 (3), p. 185-216.

Cohen-Charash, Yochi et Paul E. Spector. (2001) « The Role of Justice in Organizations : A Meta-Analysis», OrganizationalBehavior and HumanDecisionProcesses, 86,p.278-321.

Cole, S. Michael, Jeremy B. Bernerth, Frank Walter et Daniel T. Holt. (2010) « Organizational Justice and Individuals' Withdrawal : Unlocking the Influence of Emotional Exhaustion »,Journal of Management Studies, 47, p. 367-390.

Colle R. (2006), L'influence de la GRH à la carte sur la fidélité des salariés : le rôle du sentiment d'auto-détermination, Thèse de doctorat en sciences de gestion, Université Aix-Marseille III.

Colquitt J. A., 2001, « On the Dimensionality of Organizational Justice: A Construct; Validation of a Measure », Journal of Applied Psychology, 86, 3, pp. 386-400. 
Fall, Safy-Godineau et Carassus, @GRH, 2018

Colquitt, J. A., Conlon, D. E., Wesson, M. J., Porter, C. O., \& Ng, K. Y. (2001). Justice at the millennium : A meta-analytic review of 25 years of organizational justice research, Journal of Applied Psychology, 86, 425-445.

Cropanzano, R., et Ambrose, M.L. (2001). Procedural and distributive justice are more similar than you think : a monistic perspective and a research agenda. In Greenberg et Cropanzano (Eds.) Advances in Organizational Justice, (pp. 119-151), Stanford University Press : Stanford

Cropanzano, R. et Greenberg, J., (1997). Progress in organizational justice : Tunneling through the maze, International Review of Industrial and Organizational Psychology, vol.12, p. 317-372.

Crozier, M. et Friedberg E., (1977), L'acteur et le système, Editions Seuil, Paris. Vbouchon.

Dailey, R. C., \& Kirk, D. J. (1992). Distributive and procedural justice as antecedents of job dissatisfaction and intent to turnover. Human Relations, 45,(3), 305-317.

DeConinck, J.B. \& Stilwell, C.D.(2004). Incorporatingorganizational justice, role states, pay satisfaction and supervisor in a model of turnover intention. Journal Business Reviews, $57,225-231$

Desmarais, C. et C. Jameux (2001). «L'encadrement dans les collectivités locales : de la responsabilité à la responsabilisation », Politiques et management public, $\mathrm{n}^{\mathrm{o}} 3, \mathrm{p} .101$ 119.

Danna, K. \& Griffin, R.W. (1999). Health and well-being in the workplace: a review and synthesis of the literature. Journal of Management, 25(3), 357-384.

Diener, E. (1994). Assessing subjective well-being: Progress and opportunities. Social Indicators Research, 31, 103-157.

Diener E, Suh EM, Lucas RE, Smith HE. (1999). Subjective well-being: three decades of progress. Psychol. Bull. 125:276-302

Fall, A. (2014), Justice organisationnelle, reconnaissance au travail et motivation intrinsèque : résultats d'une étude empirique, Relations industrielles/Industrial Relations, 69(4), 709731.

El Akremi A, Nasr, M.I. \& Camerman, J. (2006), Justice organisationnelle : Un modèle intégrateur des antécédents et des conséquences. In El Akremi, A., Guerrero, S. \& Neveu, J. P. (Eds.), Comportement. Organisationnel (vol. 2), Editions De Boeck, pp. 47- 90

Folger, R.,et Greenberg, J., (1985).Procedural Justice :An Interpretative Analysis of Personnel System, Research in Personnel and Human Resources Management, 3(2) 143-183. 
Fall, Safy-Godineau et Carassus, @GRH, 2018

Folger, R. et Konovsky M. A., (1989). Effects of procedural and distributive justice on reactions to pay raise decisions, Academy of Management Journal, 32(1), 115-130.

Folger, Robert G. et Russel Cropanzano. (1998). Organizational Justice and Human Resource Management. Beverly Hills, CA : Sage.Folger et Konovsky, 1989).

Gillet, N., Forest, J., Girouard, S., \& Crevier-Braud, L. (2015). Justice organisationnelle et intentions de quitter: Le rôle médiateur du bien-être eudémonique. Revue Québécoise de Psychologie, 36, 127-144.

Greenberg, J. (1990) «Organizational Justice : Yesterday, Today, and Tomorrow», Journal of Management, 16, p. 399-432.

Greenberg, J. (1987) « A Taxonomy of Organizational Justice Theories», Academy of Management Review, 12, p. 9-22.

Heponiemi, T., Kuusio, H., Sinervo, T., \& Elovainio, M. (2011). Job attitudes and well-being among public vs. private physicians : Organizational justice and job control as mediators. European Journal of Public Health, 21(4), 520-525.

Hood, C. (1991). «A public management for all seasons? », Public Administration, vol. 69, (1), p. 319.

Igalens, J et Roussel, P., (1998) Méthodes de recherche en gestion des ressourceshumaines, Paris : Economica.Masterson, S. S., Kyle L., Barry M. G. et Susan M. T. (2000) « Integrating Justice and Social Exchange : The DifferingEffects of FairProcedures and Treatment on WorkRelationships», Academy of Management Journal, 43, p. 738-748.

Kausto, J., Elo, A., Lipponen, J., \& Elovainio, M. (2005). Moderating effects of job insecurity in the relationships between procedural justice and employee well-being : Gender differences. European Journal of Work and Organizational Psychology, 14(4), 431-452.

Kickul, J., Lester, S.W. \& Finkl, J. (2002). Promise breaking during radical organizational change: do justice interventions make a difference? Journal of Organizational Behavior, 23, 469-488

Knight, C. P., \& Haslam, S. A. (2010). The Relative Merits of Lean, Enriched, and EmpoweredOffices: An ExperimentalExamination of the Impact of Workspace Management Strategies on Well-Being and Productivity, Journal of Experimental Psychology : Applied, 16 (2), 158-172.

Konovsky, M.A. and Pugh, S.D. (1994) Citizenship Behavior and Social Exchange. Academy of Management Journal, 37, 656-669.

Leventhal, G. S. (1980). What should be done with equity theory? New approaches to the study of fairness in social relationships. In K. J. Gergen, M. S. Greenberg, \& R. H. 
Fall, Safy-Godineau et Carassus, @GRH, 2018

Willis (Éds), Social exchange : Advances in theory and research (p. 27-55). New York, NY : Plenum Press.

Lind, E. A. and Tom Tyler (1988), The Social Psychology of Justice. New York : Plenu

Massé, R., Poulin, C., Dassa, C., Lambert, J., Bélair, S., Battaglini, A., (1998). Élaboration et validation d'un outil de mesure du bien-être psychologique : L’ÉMMBEP. Revue Canadienne de Santé Publique, 89, 352-357.

Masterson, S. S, Lewis, K., Goldman, B. M, \& Taylor, M. S. (2000). Integrating justice and social exchange: The differing effects of fair procedures and treatment on work relationships. Academy of Management Journal, 43: 738-748.

Molines M. et Fall, A. (2016), Le rôle médiateur de l'engagement au travail dans la relation entrela justice organisationnelle et les comportements de citoyenneté : le cas de la police française, Relations Indutrielles/Industrials Relations, 71,(4), p.660-689.

Monjardet, D. (1996) «Ce que fait la police», dans Sociologie de la force publique, Paris : La Découverte.

Moorman, R.H., \& Byrne, Z.S. (2005). How does organizational justice affect organizational citizenship behavior ? In J. Greenberg \& J. A. Colquitt (Eds.), Handbook of Organizational Justice (pp. 355-382). Mahwah. New Jersey : Lawrence Erlbaum Associates.

Nasr, MI., El-Akremi, A., Vandenberghe, C. (2009). Justice organisationnelle, confiance et comportements de citoyenneté: test d'un modèle multi-sources multi-cibles de l'échange social au travail. Revue de gestion des ressources humaines, 74: 3-23 p.

O'Neill, B. S., \& Mone, M. A. (1998). Investigating equity sensitivity as a moderator of relations between self-efficacy and workplace attitudes. Journal of Applied Psychology, 83(5), 805-816.

Ohana M., (2012), Perceived Organisational Support As Mediator Of Distributive Justice And Job Satisfaction: The Moderating Role Of Group Commitment, Journal of Applied Business Research, vol. 28, n 5, p. 1063-1072

Price, J.L. (1977) The Study of Turnover. Iowa State University Press, Ames.

Ragaigne, A. (2016). Management des collectivités territoriales, Gualino; Collection : Fonction Publique; ISBN : 978-2-297-04801-9;

Roussel P., Durrieu F., Campoy E., El Akremi A., (2002), Méthodes d'équations structurelles : Recherches et applications en gestion, Edition ECONOMICA, PARIS. 
Fall, Safy-Godineau et Carassus, @GRH, 2018

Rupp, D. E., \& Cropanzano, R. (2002). The mediating effects of social exchange relationships in predicting workplace outcomes from multifoci organizational justice. Organizational Behavior and Human Decision Processes, 89, 925-946.

Simon A., Fall, A., et Carassus, D., (2015), La construction d'un baromètre pour mesurer la "performance $\mathrm{RH}$ " en milieu public : une application dans le contexte local, Revue Gestion et Management Public vol. 3, $n^{\circ} 3$, p. 5-31

Wood, S., Van Veldhoven, M., Croon, M., \& De Menezes, L.M. (2012). Enriched job design high involvement management and organizational performance : The mediating roles of job satisfaction and well-being. Human Relations, 6 (4), 419-445. 